\title{
Highly anisotropic resonant dynamics in the aligned-aligned scattering of cold diatoms
}

\section{Haowen Zhou}

Department of Chemistry, Stanford University https://orcid.org/0000-0001-5068-6758

\section{William Perreault}

Stanford University https://orcid.org/0000-0003-0639-5800

\section{Nandini Mukherjee}

Stanford University https://orcid.org/0000-0003-1486-8508

Richard Zare ( $\square$ zare@stanford.edu )

Stanford University https://orcid.org/0000-0001-5266-4253

\section{Article}

Keywords: anisotropic resonant dynamics, chemical physics

Posted Date: April 9th, 2021

DOI: https://doi.org/10.21203/rs.3.rs-356225/v1

License: (9) This work is licensed under a Creative Commons Attribution 4.0 International License. Read Full License

Version of Record: A version of this preprint was published at Nature Chemistry on May 2nd, 2022. See the published version at https://doi.org/10.1038/s41557-022-00926-z. 


\title{
Highly anisotropic resonant dynamics in the aligned-aligned scattering of cold diatoms
}

\author{
Haowen Zhou, William E. Perreault, Nandini Mukherjee*, and Richard N. Zare* \\ Department of Chemistry, Stanford University, Stanford, CA 94305 \\ *Corresponding authors. Emails: nmukherj@stanford.edu, zare@stanford.edu
}

\begin{abstract}
The dynamics of a resonant oriented scattering process dominated by a single partial wave provide the most sensitive probe of the long-range anisotropic forces important to chemical reactions. Here, we control the collision temperature and geometry to probe the dynamics of the cold $(<2 \mathrm{~K})$ rotationally inelastic scattering of a pair of optically state-prepared $\mathrm{D}_{2}$ molecules. The collision temperature is manipulated by combining the strobing action of laser state preparation and detection with the velocity dispersion of the molecular beam. When the bond axes are aligned parallel to the collision velocity, the scattering rate drops by nearly an order of magnitude when collision energies $>1 \mathrm{~K}$ are removed, demonstrating a clear geometry-dependent resonance. Using partial wave analysis of the measured scattering angular distribution, we determine that an $l=2$ shape resonance originates from the collisions between a pair of aligned $\mathrm{D}_{2}$ molecules. Our experiment illustrates the strong anisotropy of the long-range quadrupolequadrupole interaction that controls the dynamic resonance for diatom-diatom collisions.
\end{abstract}




\section{Introduction}

Long-range molecular forces are a subject of intense interest because of their importance in initiating chemical reactions. ${ }^{1}$ However, optical spectroscopy, which so effectively probes the deeply bound molecular states, cannot generally probe the most loosely bound states associated with the long-range part of the potential. As a result, its experimental interrogation remains challenging. Fortunately, low energy scattering experiments are sensitive to weak, long-range molecular forces, but their small cross sections often cause experimental noise that masks important detail. Dynamical resonances, which represent positive energy quasi-bound states of the collision complex, enhance the scattering probability in this interaction range. Several crossed-beam and merged beam techniques have demonstrated the ability to tune the collision energy in the low-energy regime and capture the increase in cross section that accompanies a scattering resonance. Both orbiting ${ }^{2-8}$ and Feshbach ${ }^{9}$ resonances were detected using various collision pairs. While these remarkable experiments were able to detect scattering resonances, they provided only limited direct information on the dynamics of the resonant collision complex.

Recently, a few experimental studies have begun to illustrate the dynamics of a scattering resonance by simultaneously measuring the scattering angular distribution. ${ }^{10-15}$ In the presence of a resonance, the scattering is dominated by a single incoming orbital or partial wave that produces a finite number of scattered orbitals. The interference of the limited number of outgoing orbitals generates a characteristic structure in the scattering angular distribution that can be used as a fingerprint of the resonance. ${ }^{11,16}$ As a result, the quasi-bound states, which are formed within the centrifugal barrier of the orbital angular momentum, provide an extremely sensitive probe for the long-range interactions. ${ }^{2}$ However, the sensitivity of these measurements is limited by any averaging over internal molecular quantum states. To completely understand how each orbital is scattered by the interaction potential as energy is exchanged between different degrees of freedom, it is essential to eliminate averaging by fully defining the internal energy states in the incoming and outgoing channels.

However, even controlling the temperature and internal energy is insufficient to map the inherently anisotropic electrostatic force fields that drive molecular processes. To fully explore the rich dynamics of diatom-diatom interactions experimentally, we must also control the collision geometry, ${ }^{17}$ which requires the selection of the $m$ state distribution within a single rovibrational energy level for each partner. ${ }^{18}$ A large number of theoretical studies have 
illustrated in great detail the importance of the relative alignment of the colliding pair on the dynamics of cold diatom-diatom scattering. ${ }^{18-26}$ Unfortunately, the vast majority of experimental work thus far has not explored the effect of the spatial alignment of the collision partners on the scattering process, and so there is very little data available for comparison with theory. Defining the initial state with this level of precision is equivalent to setting the boundary conditions for experimentally solving the dynamical equations. A scattering experiment probing the dynamical resonance with complete control of both the internal and external degrees of freedom will provide invaluable data elucidating the long-range anisotropic forces so important to chemistry. Completely alignment-controlled diatom-diatom scattering experiments have been greatly anticipated by theoreticians and experimentalists alike and might be considered one of the holy grails of scattering studies.

We previously conducted a series of quantum state controlled cold scattering experiments that made significant progress toward the goal of complete control. ${ }^{27-29}$ These experiments used control over molecular alignment to demonstrate remarkable stereodynamic effects, which likely resulted from dynamic resonances. However, in these experiments the presence of a scattering resonance was only speculated, not directly proven, because the collision energy was not tuned. Additionally, we have previously only been able to control the alignment of one of the partner molecules, partially obscuring the effects of the anisotropic collision forces on the scattering dynamics.

Here, we report a quantum-controlled collision experiment that probes the dynamics of a scattering resonance by manipulating the molecular alignment relative to the collision velocity as well as the collision energy. In these experiments, we prepared $\mathrm{D}_{2}$ molecules in a rovibrationally excited, aligned quantum state $(v=2, j=2, m)$, and studied the $\Delta j=2$ rotational relaxation $\mathrm{D}_{2}(v$ $=2, j=2, m) \rightarrow\left(v=2, j^{\prime}=0\right)$ within a supersonically expanded pure beam of $\mathrm{D}_{2}$ molecules. Here, $v$ and $j$ give the vibrational and rotational quantum numbers respectively, while $m$ gives the projection of the internal angular momentum on a chosen quantization axis. The collision temperature is manipulated by combining the strobing action of laser state preparation and detection with the velocity dispersion of the molecular beam. We show that the width of the velocity distribution of the aligned molecules contributing to the collision process is decreased by increasing the delay between the preparation and probe lasers, which reduces the available collision energy by removing hotter collisions. The strong response of the scattering rate to 
changes in the collision energy suggests that the scattering is dominated by a resonance involving a pair of rotationally aligned $\mathrm{D}_{2}(v=2, j=2)$ molecules. Using partial wave analysis of the measured angular distributions we confirm that the dynamic resonance is formed within the centrifugal barrier of the incoming partial wave with angular momentum $l=2$. Our analysis shows that the long-range quadrupole-quadrupole interaction between a pair of rotating molecules contributes significantly to this dynamical resonance.

By aligning the bond axes of both colliding partner molecules either parallel or perpendicular to the approach direction, we control the collision geometry at the molecular level. We show that the resonance behavior sensitively depends on the collision geometry, revealing the strongly anisotropic character of the molecular forces involved. In fact, the anisotropic effect is so dramatic that the inelastic scattering can be very nearly switched off by aligning both bond axes parallel to the collision velocity. The anisotropy in the quadrupole-quadrupole interactions we report here parallels earlier measurements on the dipole-dipole scattering between two electric field oriented ultracold KRb molecules, ${ }^{30}$ which demonstrated dramatic changes in the reaction barrier for the head-to-tail versus side-on orientations.

\section{Experimental results and analysis}

To study the $\Delta j=2$ rotational relaxation, pure $\mathrm{D}_{2}$ is adiabatically cooled by supersonic expansion from an Even-Lavie pulsed valve ${ }^{31}$ and collimated (divergence $12 \mathrm{mRad}$ ) into a molecular beam by a skimmer. The collimated beam defines the direction of the collision velocity in the lab frame. The $\mathrm{D}_{2}$ molecules will be aligned with respect to this axis, thus fully controlling the collision geometry at the molecular level. Nearly the complete $(>95 \%) \mathrm{D}_{2}(v=0$, $j=0)$ population is pumped into the rovibrationally excited $\mathrm{D}_{2}(v=2, j=2)$ state using Starkinduced adiabatic Raman passage (SARP). ${ }^{32}$ SARP prepares the quantum state using a pair of partially overlapping nanosecond laser pulses intercepting the $\mathrm{D}_{2}$ beam at right angles. ${ }^{33-35}$ The $\Delta j=2$ rotationally relaxed $\mathrm{D}_{2}\left(v=2, j^{\prime}=0\right)$ scattering product is detected by $(2+1)$ resonance enhanced multiphoton ionization (REMPI) with tunable nanosecond UV laser pulses. The angular distributions of rotationally relaxed $\mathrm{D}_{2}\left(v=2, j^{\prime}=0\right)$ molecules are determined from the measured time-of-flight distributions. The scattering signal is maximized when the REMPI spot is centered on the moving volume of SARP molecules (see Fig. 1 below). This is because the $\Delta j=2$ rotational relaxation produces $\mathrm{D}_{2}\left(v=2, j^{\prime}=0\right)$ molecules with $\sim 800 \mathrm{~m} / \mathrm{s}$ of translational 
energy in the moving reference frame, and so the scattering products are rapidly deflected out of the beam after they are produced. As a result, only those collisions that occur within the REMPI volume are detected, which we have experimentally verified by rastering the REMPI laser spot along the molecular beam axis.

The rotational relaxation of $\mathrm{D}_{2}(v=2, j=2)$ in the pure $\mathrm{D}_{2}$ beam can be mediated by either another state-prepared $\mathrm{D}_{2}(v=2, j=2)$, or by an unprepared $\mathrm{D}_{2}(v=0, j=0,1,2,3)$ in the ground vibrational level. Using (2+1) REMPI, the rotational population distribution of the $v=0$ ground state of $\mathrm{D}_{2}$ in the beam before state preparation was measured to be $38 \%$ in $j=0,36 \%$ in $j=1,24 \%$ in $j=2$, and $2 \%$ in $j=3$. We note that nearly all the $(v=0, j=0)$ molecules are pumped to the $(v=2, j=2)$ state by SARP, leaving primarily the $(v=0, j=1,2)$ molecules as potential unprepared collision partners.

As elaborated in our earlier work, SARP controls the bond axis alignment by selecting specific $m$ states within the rovibrational $(v, j)$ eigenstate. ${ }^{36}$ To study the stereodynamics, two specific bond axis alignments, referred to as HSARP and VSARP, were prepared by polarizing both the optical fields of the pump and Stokes laser pulses either parallel or perpendicular to the molecular beam axis. ${ }^{27}$ In HSARP the bond axis of $\mathrm{D}_{2}(v=2, j=2)$ is preferentially aligned along the collision velocity, while for the VSARP state the bond axis is aligned perpendicular. Choosing the quantization $\mathrm{z}$ axis along the collision velocity, the HSARP and VSARP states can be expressed as follows:

$$
\begin{aligned}
& |\mathrm{HSARP}\rangle \equiv \psi_{\mathrm{H}}(v=2, j=2)=|j=2, m=0\rangle \\
& |\mathrm{VSARP}\rangle \equiv \psi_{\mathrm{V}}(v=2, j=2)=-\frac{1}{2}|j=2, m=0\rangle+\sqrt{\frac{3}{8}}(|j=2, m=2\rangle+|j=2, m=-2\rangle)
\end{aligned}
$$

For simplicity we have omitted ( $v=2)$ quantum number on the right-hand side of Eqs. (1) and (2). For each of the alignments, the scattered molecules are detected at two different delays of $100 \mathrm{~ns}$ and $500 \mathrm{~ns}$ following SARP preparation. The two delays of $100 \mathrm{~ns}$ and $500 \mathrm{~ns}$ correspond to probe distances of $0.2 \mathrm{~mm}$ and $1 \mathrm{~mm}$, respectively, downstream from the SARP preparation site along the molecular beam.

Figure 1 shows the measured time-of-flight distributions of the rotationally relaxed $\mathrm{D}_{2}(v$ $=2, j^{\prime}=0$ ) for the HSARP and VSARP alignments measured at these two delays. Table I summarizes the scattering rates for HSARP and VSARP alignments at these two delays. Immediately, we see that the scattering rate drops dramatically for the HSARP alignment when 
the delay is increased from 100 to $500 \mathrm{~ns}$, while it drops much more mildly for the VSARP alignment. In fact, the HSARP scattering rate at $500 \mathrm{~ns}$ is so weak that we were unable to collect sufficient data to overcome noise and generate a clean angular distribution. Crucially, we measured that the molecular beam intensity remained nearly constant over the $0.8 \mathrm{~mm}$ displacement between the $100 \mathrm{~ns}$ and $500 \mathrm{~ns}$ probe sites.

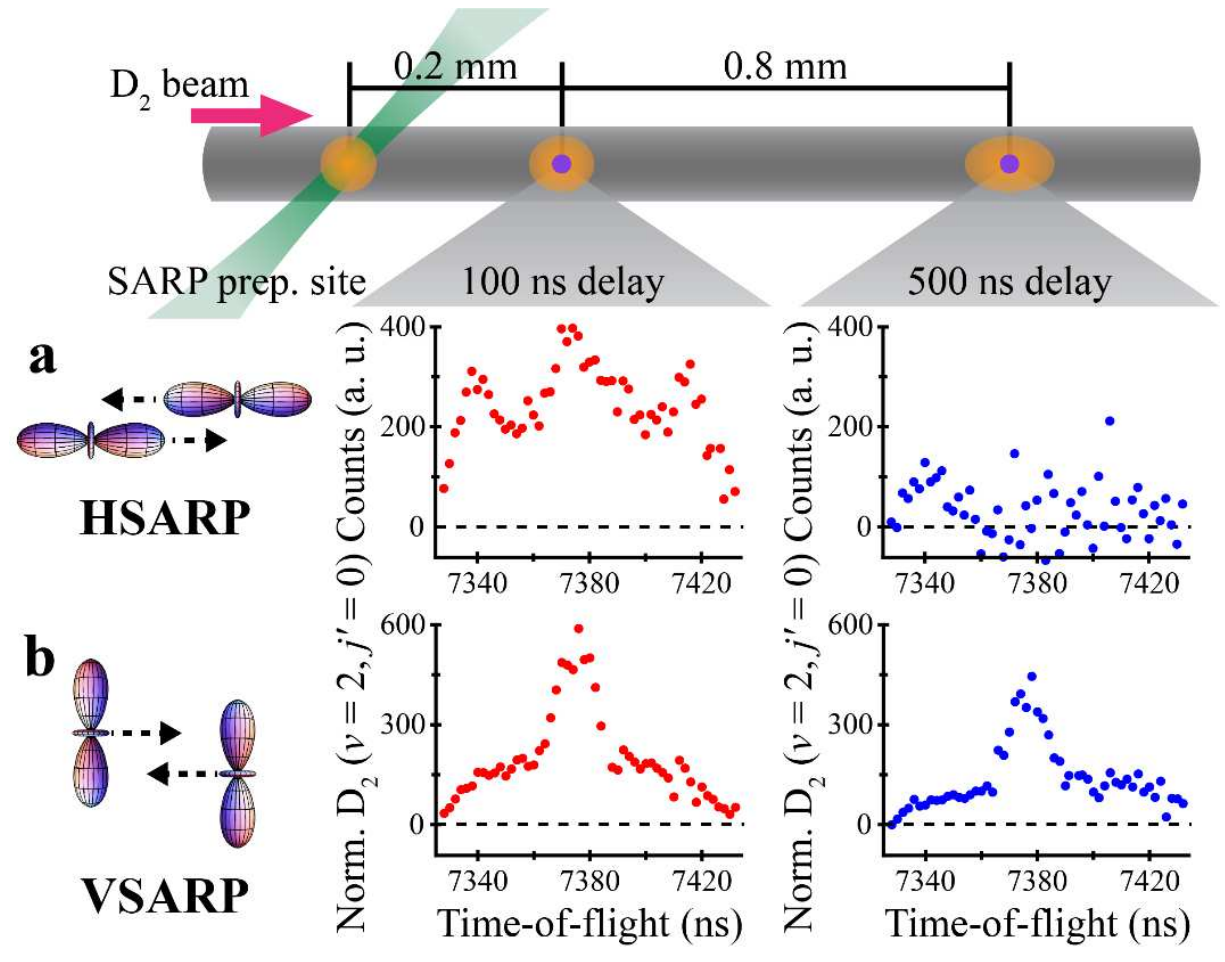

Figure 1. Measured time-of-flight distributions of scattered $\mathrm{D}_{2}\left(v=2, j^{\prime}=0\right)$ for the HSARP (a) and VSARP (b) alignments at delays of $100 \mathrm{~ns}$ (red dots) and $500 \mathrm{~ns}$ (blue dots) after SARP preparation. In HSARP, the total number of counts drops by $~ 90 \%$, while for VSARP it drops by only $\sim 40 \%$. (See Table I) The negative points for HSARP at $500 \mathrm{~ns}$ result from the signal level falling below the stochastic fluctuations of the background noise and should be taken only as a demonstration of the dramatic reduction in relaxation rate. Schematic at top shows the SARP preparation of a defined region of the molecular beam (orange spot). As the delay is increased, this spot propagates downstream and spreads out. As described in the text, only those scattering products within the small REMPI volume (purple spots) are detected. 
Table I Scattering rates as a function of SARP-REMPI delay and bond axis alignment.

\begin{tabular}{|c|c|c|}
\hline $\begin{array}{c}\mathrm{D}_{2}(v=2, j=2) \\
\text { bond-axis alignment }\end{array}$ & $\begin{array}{c}\text { Scattered } \mathrm{D}_{2}\left(v=2, j^{\prime}=0\right) \\
\text { counts/hour } \\
\text { at } 100 \mathrm{~ns} \text { delay }\end{array}$ & $\begin{array}{c}\text { Scattered } \mathrm{D}_{2}\left(v=2, j^{\prime}=0\right) \\
\text { counts/hour } \\
\text { at } 500 \text { ns delay }\end{array}$ \\
\hline HSARP & 2000 & 270 \\
\hline VSARP & 1700 & 1000 \\
\hline
\end{tabular}

To determine what change in the initial conditions of the scattering process caused the dramatic change in the scattering rate shown in Fig. 1 between 100 ns and 500 ns, we examined the collision velocity distributions at the two delays. To that end, the velocity distributions of both the prepared and unprepared $\mathrm{D}_{2}$ molecules were measured using time-of-flight mass spectrometry following $(2+1)$ REMPI at the two delays. We find that for the SARP prepared molecules the $1 / e$ width of the distribution reduces from $82 \pm 1 \mathrm{~m} / \mathrm{s}$ to $60 \pm 3 \mathrm{~m} / \mathrm{s}$ as we move from 100 to $500 \mathrm{~ns}$ delay, while for the unprepared molecules it remains constant at $89 \pm 3 \mathrm{~m} / \mathrm{s}$. The individual velocity distributions are given in Figs. 2a and b. Clearly, we observe considerable narrowing of the velocity distribution for the SARP prepared $\mathrm{D}_{2}(v=2)$ at $500 \mathrm{~ns}$. The reduction in the velocity distribution of the state-prepared molecules with increasing delay is the only change we measure in the initial conditions present for our scattering. This velocity narrowing is therefore clearly correlated to the dramatic changes in the measured scattering rate shown in Table I, and so we must understand the velocity narrowing process in order to explain the observed changes.

To understand this narrowing effect, we must consider the fact that both SARP preparation and REMPI detection take place within the narrow confines of the focused laser spots on the molecular beam, which correspond to an observation time of a few nanoseconds in the moving frame of reference. Because SARP transfers the ground-state $\mathrm{D}_{2}(v=0, j=0)$ molecules to the $(v=2, j=2)$ state within the $\sim 100 \mu \mathrm{m}$ SARP laser spot irrespective of their velocities, it sets a time $t=0$ for the state-prepared $\mathrm{D}_{2}(v=2, j=2)$ molecules (orange spots in the schematics of Figs. 1 and 2). Afterward, the state-prepared $\mathrm{D}_{2}$ with different velocities will disperse in space along the molecular beam propagation direction as shown by the elongation of the orange spots in the schematics as the delay is increased. Detection by the REMPI laser $(\sim 20$ 
$\mu \mathrm{m}, 5 \mathrm{~ns}$ ), which is shown by the purple spot in the schematics, acts like a stroboscope that sets a narrow time window for the SARP prepared $\mathrm{D}_{2}$ molecules to arrive at the probe location. This selects an increasingly narrow velocity group as the detection location is moved farther away from the SARP preparation site.

Thus, the SARP preparation and REMPI probing act as a gating mechanism, removing the fastest and slowest velocities from the distribution of the state-prepared molecules within the small volume that we probe as the molecules travel further from the preparation site. This is demonstrated by the contrast between the two solid curves in Figs. $2 \mathrm{a}$ and b. The hottest collisions occur between molecules in the opposite tails of the velocity distributions. Therefore, the available collision energy will be reduced for aligned-aligned collisions as the delay is increased because the distribution of both partners is narrowed. To be precise, $85 \%$ of scattering events occur within the collision temperature range $0-1.7 \mathrm{~K}$ at $100 \mathrm{~ns}$ delay, whereas at $500 \mathrm{~ns}$ this range is reduced to $0-0.9 \mathrm{~K}$. However, for the unprepared $\mathrm{D}_{2}$ there is nothing that sets a time $t=0$, and so its velocity spectrum remains unchanged with delay and distance as shown by the dotted curves in Figs. 2a and 2b. As a result, the collision temperature does not significantly change for the aligned-unaligned collisions with increasing delay. In Fig. 2c, we show the collision speed for both the aligned-aligned and aligned-unaligned collision pairs at 100 and 500 ns delay, calculated by convoluting the measured distributions of the individual partners as described in the Methods section. It can be clearly seen that only the aligned-aligned collision pair at $500 \mathrm{~ns}$ shows a significant reduction in its speed distribution.

As the hottest collisions between the state prepared $\mathrm{D}_{2}(v=2)$ molecules are removed at $500 \mathrm{~ns}$ delay, thus reducing the collision temperature, the scattering rate is dramatically reduced. Recall that the measured scattering angular distributions in Fig. 1 originate only from the timegated SARP prepared $\mathrm{D}_{2}$ molecules that are in the small REMPI volume at the time of probing. Therefore, we conclude that the changes in the observed scattering rates for both alignments result from the reduction of the aligned-aligned collision temperature between the two delays. The observed rotational relaxation of $\mathrm{D}_{2}(v=2, j=2)$ is therefore caused via collision with another $\mathrm{D}_{2}(v=2, j=2)$. We determine from conservation of energy that only one of the $\mathrm{D}_{2}$ molecules in the aligned-aligned scattering pair is rotationally relaxed during the collision event. Because we do not select the nuclear spin state of the SARP prepared $\mathrm{D}_{2}$ molecules, only one sixth of the aligned-aligned scattering involves a pair of identical bosons. Furthermore, the large 
change in HSARP scattering rate in response to a relatively small change in collision temperature indicates the presence of a scattering resonance. The presence of a scattering resonance is further supported by the spectacular difference in scattering for the HSARP versus VSARP alignment.

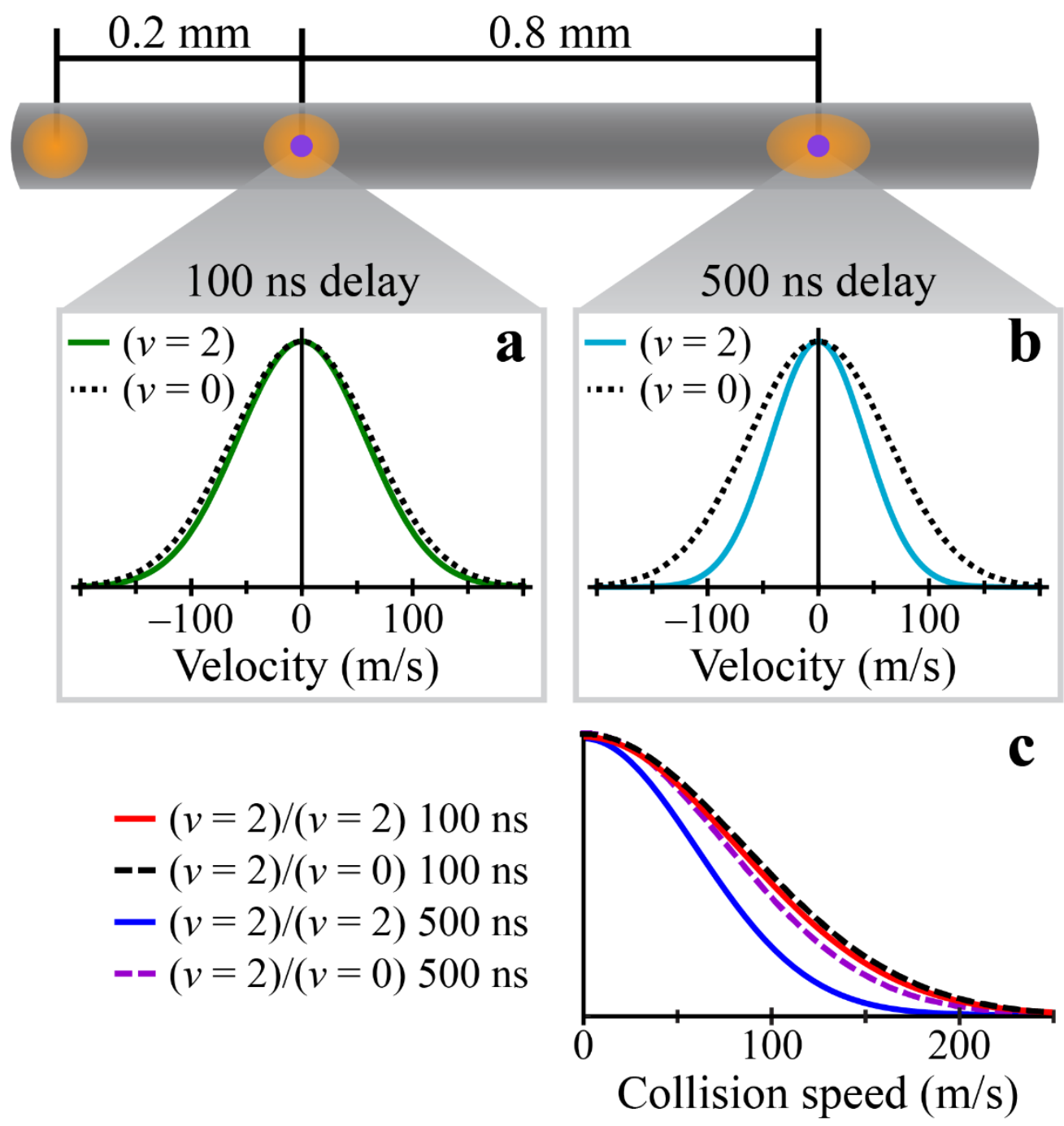

Figure 2. (a) The velocity distributions of the prepared $\mathrm{D}_{2}(v=2, j=2)$ (green curve) and unprepared $\mathrm{D}_{2}(v=0, j=1,2)$ (black dotted curve) at $100 \mathrm{~ns}$ delay. (b) The velocity distributions of the prepared $\mathrm{D}_{2}(v=2, j=2)$ (cyan curve) and unprepared $\mathrm{D}_{2}(v=0, j=1,2)$ (black dotted curve) at $500 \mathrm{~ns}$ delay. All velocities are determined by fitting the time-of-flight distribution taking into account the electron recoil, which gives a measurement error of less than $3 \mathrm{~m} / \mathrm{s}$. (c) Collision speeds for both the aligned-aligned (red and blue curves) and aligned-unaligned (black and magenta dashed curves) collision pairs at the two delays. As described in the Methods section, these curves are generated by convoluting the velocity distributions given in (a) and (b). The only substantial change is in the collision speed for the aligned-aligned collisions at $500 \mathrm{~ns}$ (solid blue curve). All three other curves are within our measurement error. 
To further understand the resonance character of the $\Delta j=2$ relaxation, we carried out a partial wave analysis of the angular distribution of the scattered $\mathrm{D}_{2}\left(v=2, j^{\prime}=0\right)$ at 100 ns delay for the HSARP and VSARP alignments. Our partial wave analysis is a purely phenomenological approach that uses the basic conservation principles of energy, momentum, angular momentum, and parity to determine the relative contributions of the outgoing partial waves. The partial wave fitting of the measured angular distributions for the two axis alignments are shown below in Fig. 4. By determining the relative amplitudes of the outgoing orbitals and correlating each outgoing orbital with each incoming one, the partial wave analysis allows us to extract information on the dynamics of the rotationally inelastic collision. At resonance, a single partial wave dominates the scattering process, giving rise to a characteristic angular distribution that results from the interference of a small number of outgoing or scattered partial waves.

The scattering angular distributions can be expressed as follows: ${ }^{27-29}$

$$
\frac{\mathrm{d} \sigma}{\mathrm{d} \theta}=\sin \theta \int_{0}^{2 \pi}\left|\sum_{m} a_{m} q_{j=2, m \rightarrow j^{\prime}=0}\right|^{2} \mathrm{~d} \varphi,
$$

where $q_{j=2, m \rightarrow j^{\prime}=0}$ represents the probability amplitude to find the $\mathrm{D}_{2}\left(v=2, j^{\prime}=0\right)$ scattered from the initial state $|j=2, m\rangle$ within a differential solid angle $d \Omega$ in a direction defined by the polar angle $\theta$ and azimuthal angle $\varphi . a_{m}$ gives the amplitude of the various initial $|j=2, m\rangle$ states, which appear in the expressions for the HSARP and VSARP states given in Eq. (1) and (2). Because the angular distribution results from the coherent superposition of the scattered orbitals, the scattering amplitude in Eq. (3) can be written as:

$$
q_{j=2, m \rightarrow j^{\prime}=0}=\sum_{l^{\prime}, m_{l^{\prime}}} c_{l^{\prime} m_{l^{\prime}}} \mathrm{Y}_{l^{\prime} m_{l^{\prime}}}(\theta, \varphi)
$$

In Eq. (4), the complex expansion coefficient $c_{l^{\prime} m_{l^{\prime}}}$ gives the amplitude for the outgoing orbit $l^{\prime} m_{l^{\prime}}$, which is represented by the spherical harmonic $\mathrm{Y}_{l^{\prime} m_{l^{\prime}}}(\theta, \varphi)$ 


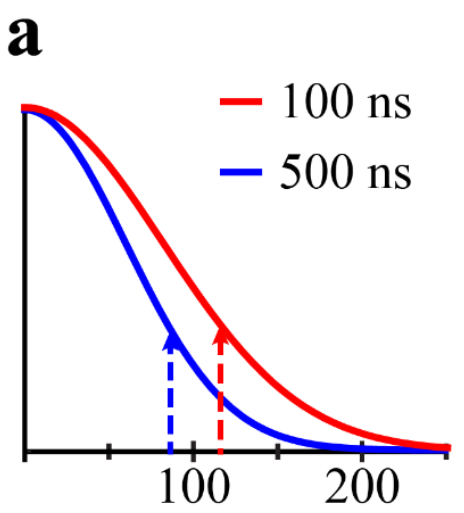

Collision speed $(\mathrm{m} / \mathrm{s})$

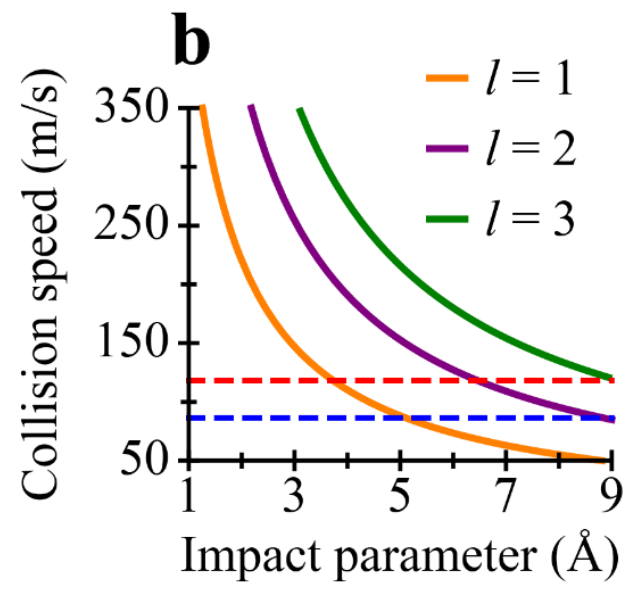

Figure 3. (a) The collision speed distribution for the $\mathrm{D}_{2}(v=2) / \mathrm{D}_{2}(v=2)$ collision pair at $100 \mathrm{~ns}$ (solid red) and $500 \mathrm{~ns}$ delay (solid blue). The red and blue dashed lines show the collision velocity range containing $85 \%$ of the $\mathrm{D}_{2}(v=2) / \mathrm{D}_{2}(v=2)$ collisions at 100 and $500 \mathrm{~ns}$ delays, respectively. (b) Contour lines for the orbital angular momenta $l=1$ (solid orange), $l=2$ (solid purple), $l=3$ (solid green) as a function of the collision speed and impact parameter. The red and blue dashed lines give the upper limit of the collision speed range for the delays of $100 \mathrm{~ns}$ and $500 \mathrm{~ns}$ corresponding to the dashed lines in (a).

To determine the outgoing orbitals $l^{\prime} m_{l^{\prime}}$ from the incoming orbitals using the conservation laws, we must first determine the incoming orbitals $l$ involved in the cold collision. To do this, we make use of the collision speed distributions shown in Fig. 3. Figure 3b shows the orbital angular momentum contours as a function of the collision speed $u_{c}$ and the impact parameter $b$ generated using the semi-classical expression $u_{c}=\sqrt{l(l+1)} \mathrm{h} / \mu b$. As shown by Fig. $3 \mathrm{~b}$, all partial waves with $l \geq 3$ have impact parameters greater than the range of the $\mathrm{H}_{2}-\mathrm{H}_{2}$ scattering potential ${ }^{37}$ for all relative velocities present. Therefore, for the collision speed distributions presented in Figs. 2 and 3, only the incoming orbitals $l=0,1,2$ contribute to the scattering process. From conservation, we can then determine that only outgoing waves $l^{\prime}=0-6$ need to be considered in our fits of the scattering angular distribution. Using the restricted set of outgoing waves, we were able to determine the complex amplitudes $c_{l^{\prime} m^{\prime}}$ for the outgoing orbitals $l^{\prime}$ by numerically fitting the measured HSARP and VSARP angular distributions using the expression given in Eq. (3). The contributions of both positive and negative collision velocities were included in the analysis for the symmetric collision velocity distribution (Fig. 2). 

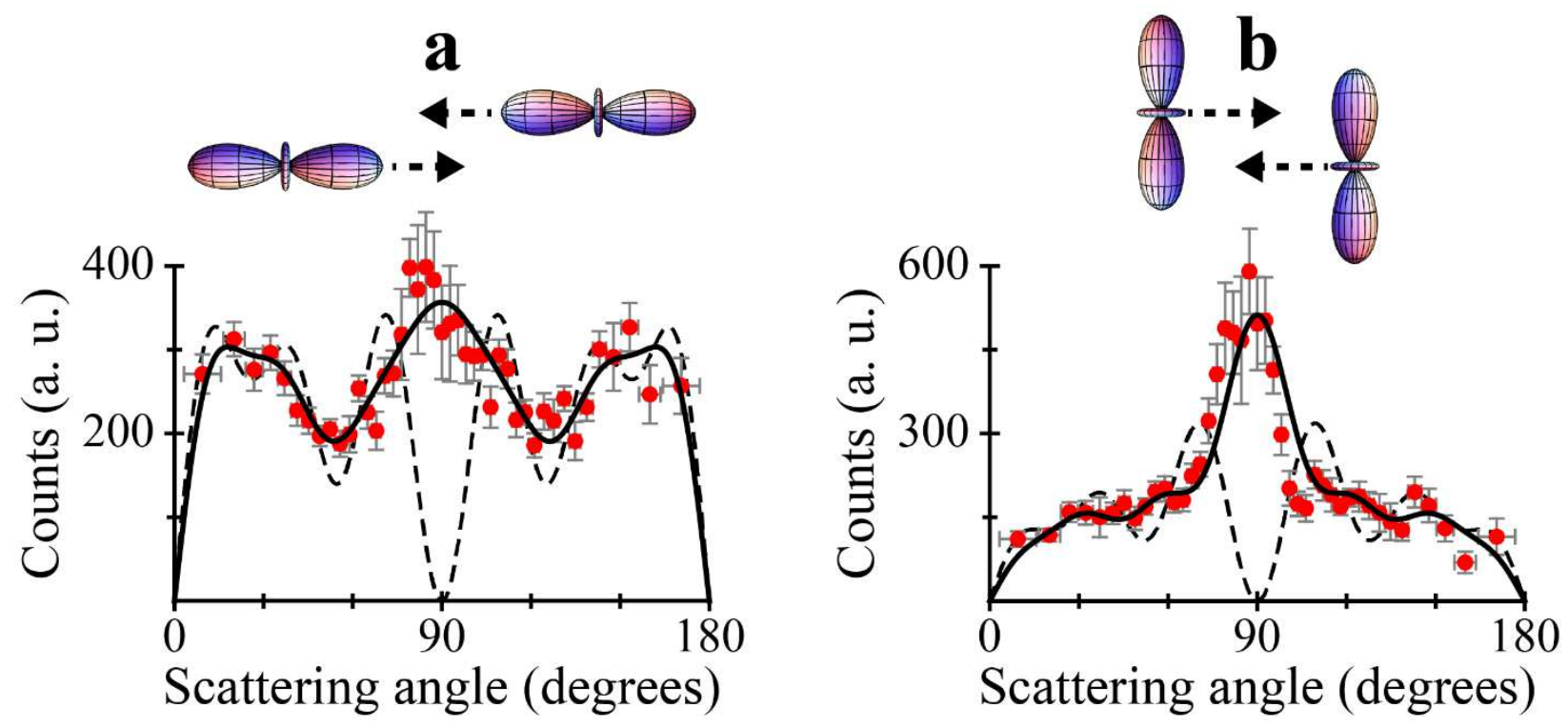

Figure 4. Experimentally measured scattering angular distributions and fits using partial wave analysis for HSARP (a) and VSARP (b) bond-axis alignments. The red dots represent experimental data taken at $100 \mathrm{~ns}$ delay. The solid and dashed black curves represent fits using partial wave analysis. As described in the main text, the solid black curve gives the fit produced using even outgoing orbitals $l^{\prime}=0,2,4,6$, whereas the dashed curve gives the fit produced using odd outgoing orbitals $l^{\prime}=1,3,5$. The error bars represent the standard deviation in each time-of-flight bin.

In Fig. 4 we show that only the even outgoing orbitals $l^{\prime}=0,2,4,6$ produce good fits of the HSARP and VSARP angular distributions (solid curves), yielding an $\mathrm{R}^{2}=0.99$ for HSARP and $\mathrm{R}^{2}=0.96$ for VSARP. In contrast, the odd outgoing orbitals $l^{\prime}=1,3,5$ did not fit either the HSARP or VSARP angular distributions well, as shown by the dashed curves in Fig. 4. The selection rule for the $\Delta j=2$ transition is determined by the two leading quadrupolar terms of the expanded interaction potential: $\mathrm{U}_{202}(r) \mathrm{Y}_{2 m}\left(\theta_{1}, \varphi_{1}\right) \mathrm{Y}_{00}\left(\theta_{2}, \varphi_{2}\right) \mathrm{Y}_{2 m}{ }^{*}(\theta, \varphi)$ and $\mathrm{U}_{224}(r) \mathrm{Y}_{2 m_{1}}\left(\theta_{1}, \varphi_{1}\right) \mathrm{Y}_{2 m_{2}}\left(\theta_{2}, \varphi_{2}\right) \mathrm{Y}_{4\left(m_{1}+m_{2}\right)}^{*}(\theta, \varphi) .{ }^{19,38,39}$ Here, $\left(\theta_{1}, \varphi_{1}\right)$, and $\left(\theta_{2}, \varphi_{2}\right)$ define the alignments of the interacting diatoms in the center-of-mass frame, and $(r, \theta, \varphi)$ defines the radial vector $\hat{r}$ connecting the center-of-mass of the two molecules. The potential term $\mathrm{U}_{202}$ represents the anisotropic interaction that leaves the rotational state of the partner unchanged and is therefore similar to the anisotropy that drives $\Delta j=2$ transitions in atom-diatom collisions. The quadrupole-quadrupole interactions where both molecular rotations are coupled result exclusively from $\mathrm{U}_{224}$, which has the largest long-range contribution. These quadrupolar interactions lead to the selection rule $\Delta l=0, \pm 2, \pm 4$ for the orbitals. As a result, the parity is 
conserved in the scattering process, meaning that even outgoing orbitals arise selectively from the scattering of even incoming orbitals. Therefore, the exclusive fit of the scattering distributions with the even outgoing orbitals $l^{\prime}=0,2,4,6$ suggests that only the even incoming orbitals $l=0,2$ are involved in the cold scattering. In contrast, the failure to fit the angular distributions with the odd outgoing orbitals $l^{\prime}=1,3,5$ indicates a negligible contribution from the incoming orbital $l=1$.

A scattering resonance is identified when the outgoing orbitals correlate dominantly to a single incoming orbital. In Table II below, we present the relative amplitudes of the even outgoing orbitals determined from the fits of the HSARP data. We choose to examine these amplitudes because the scattering process for the HSARP preparation goes from a single incoming state $(v=2, j=2, m=0)$ to a single outgoing state $\left(v=2, j^{\prime}=0, m^{\prime}=0\right)$, and so the partial wave analysis involves fewer coefficients so that the fitting is most reliable. Additionally, the measured temperature dependence and therefore resonance behavior (Fig. 1) is significantly stronger for the HSARP alignment.

Table II. Amplitudes of outgoing orbitals, correlated incoming orbitals, and associated potential terms that connect the two for the HSARP alignment determined from our even wave fit of the angular distribution as described in the text and shown in Fig. 4 a.

\begin{tabular}{|c|c|c|}
\hline $\begin{array}{c}\text { Outgoing Orbital } \\
\mathrm{Y}_{l^{\prime} m_{l^{\prime}}}(\theta, \varphi)\end{array}$ & $\begin{array}{c}\text { Amplitude of } \\
\text { outgoing orbital } \\
c_{l^{\prime} m_{l^{\prime}}}\end{array}$ & \begin{tabular}{c} 
correlating incoming orbital \\
\hline $\mathrm{Y}_{00}$
\end{tabular} \\
\hline $\mathrm{Y}_{20}$ & 0.17 & $l=0$ via $\mathrm{U}_{202}, \boldsymbol{l}=\mathbf{2}$ via $\mathbf{U}_{\mathbf{2 0 2}}$ \& $\mathbf{U}_{\mathbf{2 2 4}}$ \\
\hline $\mathrm{Y}_{40}$ & $0.23 \exp [i 0.8]$ & $l=0$ via $\mathrm{U}_{224} \boldsymbol{l}=\mathbf{2}$ via $\mathbf{U}_{\mathbf{2 0 2}} \mathbf{\&} \mathbf{U}_{\mathbf{2 2 4}}$ \\
\hline $\mathrm{Y}_{60}$ & $0.2 \exp [i 1.6]$ & $\boldsymbol{l}=\mathbf{2}$ via $\mathbf{U}_{\mathbf{2 2 4}}$ \\
\hline
\end{tabular}

Table II shows that the outgoing orbital $l^{\prime}=0$ has the largest amplitude, and it arises exclusively from the scattering of the input orbital $l=2$ by the anisotropic interaction $\mathrm{U}_{202}$. We note that the outgoing $l^{\prime}=6$ orbital is generated exclusively from the scattering of the incoming $l$ 
$=2$ orbital by the long-range quadrupole-quadrupole interaction $\mathrm{U}_{224}$. In contrast, the scattered orbitals $l^{\prime}=2,4$ include contributions from both $l=0$ and 2 via the long-range interactions $\mathrm{U}_{202}$ and $\mathrm{U}_{224}$. Table II shows that the scattered orbitals dominantly correlate to the incoming $l=2$ or $d$-wave that faces a centrifugal barrier to low energy scattering. The strong scattering of the $d$ wave indicates excitation of a quasi-resonant $\mathrm{D}_{2}-\mathrm{D}_{2}$ collision complex contained within the centrifugal barrier of the $l=2$ orbital. An order of magnitude reduction of the HSARP scattering with increasing delay clearly suggests that this quasi-resonant state is exclusively accessible to the hot collision pairs that were present at $100 \mathrm{~ns}$ but removed at $500 \mathrm{~ns}$.

To explain the temperature tuning of the observed $l=2$ shape resonance, we return to the speed distribution for $\mathrm{D}_{2}(v=2) / \mathrm{D}_{2}(v=2)$ collisions at $100 \mathrm{~ns}$ and $500 \mathrm{~ns}$ delays presented in Fig. 3a. Figure 3a shows that for the speed distribution of $\mathrm{D}_{2}(v=2) / \mathrm{D}_{2}(v=2)$ collisions at 100 ns (solid red curve), $85 \%$ of the collisions occur with a speed $u_{c} \leq 118 \mathrm{~m} / \mathrm{s}$ as indicated by the red dashed line, whereas at $500 \mathrm{~ns}$ (solid blue curve) the range shrinks to $u_{c} \leq 86 \mathrm{~m} / \mathrm{s}$. Figure $3 \mathrm{~b}$ shows that for the collision speed distribution at $100 \mathrm{~ns}$, an impact parameter $b \geq 6 \AA$ is required to support the $l=2$ orbital. In contrast, an impact parameter $b \geq 8 \AA$ is required for the range of collision speed $u_{c} \leq 86 \mathrm{~m} / \mathrm{s}$ at $500 \mathrm{~ns}$. The large impact parameter required for the $l=2$ shape resonance at $500 \mathrm{~ns}$ delay falls at the very edge of the $\mathrm{D}_{2}-\mathrm{D}_{2}$ interaction range, ${ }^{37}$ dramatically reducing the scattering probability. This qualitatively explains the weaker scattering rate experimentally observed at this delay.

Figure 3 also shows that the collision speed range at both $100 \mathrm{~ns}$ and $500 \mathrm{~ns}$ support $l=1$ scattering. However, using the partial wave analysis of the measured angular distribution, we found no significant contribution from this orbital. According to an earlier theoretical calculation on $\mathrm{HD}-\mathrm{H}_{2}$ scattering, ${ }^{40}$ the $l=1$ resonant complex represents a bound state below the $\mathrm{HD}-\mathrm{H}_{2}$ dissociation limit, and therefore it is not accessible by the colliding pair, which agrees with the result of our partial wave analysis.

\section{Discussion and Conclusions}

We have demonstrated that low temperature scattering is extremely sensitive to the collision geometry at the molecular level. By aligning the bond axes of the collision pair either parallel 
(HSARP) or perpendicular (VSARP) to the collision velocity, we observe remarkably different angular distributions as well as responses of the scattering rates to changes in the collision energy. The HSARP scattering rate, which is comparable to the VSARP rate at $100 \mathrm{~ns}$, falls by nearly a factor of 10 with decreasing collision temperature at $500 \mathrm{~ns}$. Our measurement shows that the drastic reduction of the scattering rate for the HSARP geometry with increasing delay is caused by a dynamical resonance that is only accessible to the hot collisions available at $100 \mathrm{~ns}$. The VSARP scattering rate reduces much less as the collision temperature is decreased. Nonetheless, the fact that the scattering angular distribution for VSARP retains the exact same shape at $500 \mathrm{~ns}$ delay suggests that the same resonance is still present for this geometry but that it can be accessed by somewhat colder collisions. Thus, our experiment demonstrates that the resonance behavior depends sensitively on the collision geometry for this diatom-diatom scattering system. This illustrates the strong anisotropy of the long-range quadrupole-quadrupole interaction that controls the dynamic resonance, suggesting that these sorts of anisotropic effects will likely be present in other diatom-diatom collisions. To our knowledge, the anisotropy of molecular resonance behavior has not previously been experimentally measured with this level of detail.

The observation of the dynamics of a collision process involving a pair of aligned molecules was made possible by utilizing the stroboscopic effect of the laser state preparation and delayed probing with nanosecond pulses. As we have shown here, the laser gating mechanism allowed us to control the distribution of collision speed between a pair of state prepared molecules. By changing the alignment of both bond axes of the colliding partners with respect to their approach direction, we observe a dramatic difference in the response of their scattering rate to the collision temperature. By combining alignment and temperature control, we could simultaneously locate and fingerprint a resonance experimentally. The aligned-aligned scattering reported here is among the first of its kind, approaching the experimental ideal of complete control over both the internal and external degrees of freedom. The nearly complete control that we have achieved here breaks new ground in the understanding of the geometric dependence of a dynamic resonance, which has important implications in cold and ultracold four-center collision processes. ${ }^{41}$ 


\section{Materials and Methods}

$\mathrm{D}_{2}$ molecules (Cambridge Isotope Labs, 99.8\%) at a stagnation pressure of $250 \mathrm{psi}$ are supersonically expanded and collimated to form a molecular beam (divergence $12 \mathrm{mRad}$ ) using a narrow $(600 \mu \mathrm{m})$ skimmer placed at a distance of $\sim 10 \mathrm{~cm}$ from the pulsed valve. The $\mathrm{D}_{2}(v=0, j$ $=0)$ are transferred to the $(v=2, j=2, m)$ state using a coherent optical process called Starkinduced adiabatic Raman passage (SARP). SARP accomplishes population transfer between a pair of rovibrational states using two-photon excitation with far-off resonant intermediate states. To transfer the complete population to a target state, SARP manipulates the crossings of the optically dressed adiabatic states using a pair of partially overlapping phase coherent nanosecond laser pulses. The dynamic Stark shift from the intense laser pulses controls the crossing of resonance. The efficiency of adiabatic population transfer relies on a slow sweeping rate of the Stark-induced detuning and a strong two-photon Rabi frequency that lifts the degeneracy of the adiabatic states at the crossing. To transfer the complete population of $\mathrm{D}_{2}(v=0, j=0)$ to $\mathrm{D}_{2}(v=$ $2, j=2, m)$ we have used a strong pump pulse (10 ns, $\left.1064 \mathrm{~nm}, 100 \mathrm{~J} / \mathrm{mm}^{2}\right)$ partially overlapping with a weaker Stokes pulse $\left(5 \mathrm{~ns}, 655 \mathrm{~nm}, 20 \mathrm{~J} / \mathrm{mm}^{2}\right)$ intersecting the molecular beam transversely. The pump pulse is obtained from an injection-seeded, Q-switched $\mathrm{Nd}^{3+}: \mathrm{YAG}$ laser (PRO-290, Spectra-Physics), and the Stokes pulse is derived from a pulsed dye amplifier (PrecisionScan, Sirah) seeded by a frequency-stabilized ring dye laser (Matisse, Sirah) and pumped by the second harmonic of the pump laser. Following an optical delay of $6 \mathrm{~ns}$, the pump and Stokes beams are combined using a dichroic beamsplitter before being focused onto the molecular beam using a $50 \mathrm{~cm}$ focal length lens. The region of the molecular beam excited by SARP is experimentally measured to have a diameter of $\sim 100 \mu \mathrm{m}$. More detailed descriptions of SARP can be found elsewhere. ${ }^{32-35}$

Both the SARP prepared and scattered $\mathrm{D}_{2}$ are probed using a UV laser pulse (5 ns, 213 $\mathrm{nm}, 0.6 \mathrm{~J} / \mathrm{mm}^{2}$ ) that state selectively ionizes them by $(2+1)$ resonance enhanced multiphoton ionization (REMPI). A UV beam collimator was designed to control the probe spot size on the molecular beam. The REMPI pulse is obtained by using two different $\beta$-barium borate crystals in sequence to generate the third harmonic of a tunable pulsed dye laser (Cobra-Stretch, Sirah) pumped by a Q-switched $\mathrm{Nd}^{3+}$ :YAG laser (PL9020, Continuum Lasers, Inc.). The velocity distribution of the scattered molecules along the molecular beam axis is directly determined from the measured time-of-flight (TOF) spectrum. Because the scattering center-of-mass frame 
coincides with the moving frame of reference of the molecular beam, the scattering angular distribution can then be easily extracted from the velocity distribution. ${ }^{27}$ The probe polarization is held perpendicular to the time-of-flight axis to minimize the contribution of photoelectron recoil velocity ${ }^{42}$ to the measured time-of-flight spectrum.

To control the collision temperature, we probed the scattering at two delays, 100 and 500 $\mathrm{ns}$, following SARP preparation. For the $\mathrm{D}_{2}$ molecules, which are travelling at the supersonic speed of $\sim 2 \mathrm{~km} / \mathrm{s}$, these delays correspond to distances of $0.2 \mathrm{~mm}$ and $1 \mathrm{~mm}$ downstream from the SARP preparation site (Fig. 1). Because the state preparation and detection take place within the narrow confines of the focused laser spots that last only for a few nanoseconds, this acts like a stroboscope selecting the narrow velocity group that is able to reach the probed volume in a given time. The velocity distribution of SARP prepared $\mathrm{D}_{2}$ is therefore squeezed within the small REMPI probe volume as the displacement along the molecular beam axis is increased. Because we only detect scattering products generated within the REMPI spot, this laser gating action reduces the collision temperature in the moving frame of the molecular beam. Using $(2+1)$ REMPI we recorded the velocity spectra of the SARP prepared $\mathrm{D}_{2}(v=2, j=2)$ and unprepared $\mathrm{D}_{2}(v=0, j)$ molecules at the two probe sites. These measurements were then used to calculate the collision velocity distribution between different $\mathrm{D}_{2}$ collision pairs using the following equation: $f\left(\mathrm{v}_{c}\right)=\int_{-\infty}^{\infty} \exp \left\{-\left(x / \Delta_{1}\right)^{2}\right\} \exp \left\{-\left[\left(x-\mathrm{v}_{0}+\mathrm{v}_{c}\right) / \Delta_{2}\right]^{2}\right\}$.

Here, $\Delta_{1}$ and $\Delta_{2}$ represent the width of the individual velocity distributions of the colliding pairs, and vo gives the difference in their peak speed. For collisions between excited pairs of $\mathrm{D}_{2}$ molecules, $\Delta_{1}=\Delta_{2}$. The collision speed distribution is given by: $g\left(u_{c}\right)=f\left(u_{c}\right)+f\left(-u_{c}\right)$. The calculated collision speed distributions at the two delays are displayed in Fig. 2c for various collision pairs. To ground our scattering rate measurements, we measure the intensity of the molecular beam at the two probe sites, finding that it remained nearly unchanged within the range of distances probed. This validates our finding that the observed change in the HSARP and VSARP scattering rate arises from a resonance effect. 


\section{Acknowledgments}

This work has been supported by the U.S. Army Research Office under ARO Grant Nos. W911NF-19-1-0163 and W911NF-19-1-0283.

\section{Data availability}

Data files are available upon request from nmukherj@stanford.edu.

\section{Code availability}

Computer code for fitting is available upon request from nmukherji@stanford.edu.

\section{Author Contributions}

Experimental data were taken by HZ, WEP, and NM, and calculations were done by NM. All authors participated in discussion and the writing of the manuscript.

\section{Conflict of Interest}

The authors declare no conflicts of interest.

\section{References}

1. Weck, P. F. \& Balakrishnan, N. Importance of long-range interactions in chemical reactions at cold and ultracold temperatures. Int. Rev. Phys. Chem. 25, 283-311 (2006).

2. Naulin, C. \& Costes, M. Experimental search for scattering resonances in near cold molecular collisions. Int. Rev. Phys. Chem. 33, 427-446 (2014).

3. Henson, A. B., Gersten, S., Shagam, Y., Narevicius, J. \& Narevicius, E. Observation of Resonances in Penning Ionization Reactions at Sub-Kelvin Temperatures in Merged Beams. Science 338, 234-239 (2012).

4. García-Vela, A., Cabanillas-Vidosa, I., Ferrero, J. C. \& Pino, G. A. The role of orbiting resonances in the vibrational relaxation of $\mathrm{I}_{2}\left(\mathrm{~B}, \mathrm{v}^{\prime}=21\right)$ by collisions with He at very low energies: a theoretical and experimental study. Phys. Chem. Chem. Phys. 14, 5570 (2012). 
5. Lavert-Ofir, E. et al. Observation of the isotope effect in sub-kelvin reactions. Nat. Chem. 6, 332-335 (2014).

6. Bergeat, A., Onvlee, J., Naulin, C., Van Der Avoird, A. \& Costes, M. Quantum dynamical resonances in low-energy $\mathrm{CO}(\mathrm{j}=0)+\mathrm{He}$ inelastic collisions. Nat. Chem. 7, 349-353 (2015).

7. Jankunas, J., Jachymski, K., Hapka, M. \& Osterwalder, A. Observation of orbiting resonances in $\mathrm{He}\left({ }^{3} \mathrm{~S}_{1}\right)+\mathrm{NH}_{3}$ Penning ionization. J. Chem. Phys. 142, 164305 (2015).

8. Costes, M. \& Naulin, C. Observation of quantum dynamical resonances in near cold inelastic collisions of astrophysical molecules. Chem. Sci. 7, 2462-2469 (2016).

9. Köhler, T., Góral, K. \& Julienne, P. S. Production of cold molecules via magnetically tunable Feshbach resonances. Rev. Mod. Phys. 78, 1311-1361 (2006).

10. Wang, T. et al. Dynamical resonances accessible only by reagent vibrational excitation in the F + HD $\rightarrow \mathrm{HF}+\mathrm{D}$ reaction. Science 342, 1499-1502 (2013).

11. Vogels, S. N. et al. Imaging resonances in low-energy NO-He inelastic collisions. Science 350, 787-790 (2015).

12. Yang, T. et al. Extremely short-lived reaction resonances in $\mathrm{Cl}+\mathrm{HD}(\mathrm{v}=1) \rightarrow \mathrm{DCl}+\mathrm{H}$ due to chemical bond softening. Science 347, 60-63 (2015).

13. Onvlee, J. et al. Imaging quantum stereodynamics through Fraunhofer scattering of NO radicals with rare-gas atoms. Nat. Chem. 9, 226-233 (2016).

14. Vogels, S. N. et al. Scattering resonances in bimolecular collisions between NO radicals and H2 challenge the theoretical gold standard. Nat. Chem. 10, 435-440 (2018).

15. Paliwal, P. et al. Determining the nature of quantum resonances by probing elastic and reactive scattering in cold collisions. Nat. Chem. 13, 94-98 (2021).

16. Klein, A. et al. Directly probing anisotropy in atom - molecule collisions through quantum scattering resonances. Nat. Phys. 13, 35-38 (2017).

17. Aoiz, F. J. et al. A new perspective: imaging the stereochemistry of molecular collisions. Phys. Chem. Chem. Phys. 17, 30210-30228 (2015).

18. Schaefer, J. \& Meyer, W. Theoretical studies of $\mathrm{H}_{2}-\mathrm{H}_{2}$ collisions. I. Elastic scattering of ground state para- and ortho- $\mathrm{H}_{2}$ in the rigid rotor approximation. J. Chem. Phys. 70, 344360 (1979).

19. Buck, U., Huisken, F., Maneke, G. \& Schaefer, J. State resolved rotational excitation in 
$\mathrm{HD}+\mathrm{D}_{2}$ collisions. I. Angular dependence of $0 \rightarrow 2$ transitions. J. Chem. Phys. 74, 535-544 (1981).

20. Lee, T.-G. et al. State-to-state rotational transitions in $\mathrm{H}_{2}+\mathrm{H}_{2}$ collisions at low temperatures. J. Chem. Phys. 125, 114302 (2006).

21. Quéméner, G. \& Balakrishnan, N. Quantum calculations of $\mathrm{H}_{2}-\mathrm{H}_{2}$ collisions: From ultracold to thermal energies. J. Chem. Phys. 130, (2009).

22. Balakrishnan, N., Quéméner, G., Forrey, R. C., Hinde, R. J. \& Stancil, P. C. Fulldimensional quantum dynamics calculations of $\mathrm{H}_{2}-\mathrm{H}_{2}$ collisions. J. Chem. Phys. 134, 014301 (2011).

23. Sultanov, R. A., Guster, D. \& Adhikari, S. K. Low temperature HD + ortho-/para-H2 inelastic scattering of astrophysical interest. J. Phys. B At. Mol. Opt. Phys. 49, 015203 (2016).

24. Jambrina, P. G. et al. Stereodynamical Control of a Quantum Scattering Resonance in Cold Molecular Collisions. Phys. Rev. Lett. 123, 43401 (2019).

25. Croft, J. F. E. \& Balakrishnan, N. Controlling rotational quenching rates in cold molecular collisions. J. Chem. Phys. 150, (2019).

26. Yang, B. et al. Quantum dynamics of $\mathrm{CO}-\mathrm{H}_{2}$ in full dimensionality. Nat. Commun. 6, 1-8 (2015).

27. Perreault, W. E., Mukherjee, N. \& Zare, R. N. Quantum control of molecular collisions at 1 Kelvin. Science 358, 356-359 (2017).

28. Perreault, W. E., Mukherjee, N. \& Zare, R. N. Quantum controlled rotationally inelastic scattering of $\mathrm{HD}$ with $\mathrm{H}_{2}$ and $\mathrm{D}_{2}$ near 1 Kelvin reveals collisional partner reorientation. Nat. Chem. 10, 561-567 (2018).

29. Perreault, W. E., Mukherjee, N. \& Zare, R. N. HD $(v=1, j=2, m)$ orientation controls HD-He rotationally inelastic scattering near 1 K. J. Chem. Phys. 150, 174301 (2019).

30. Ni, K. K. et al. Dipolar collisions of polar molecules in the quantum regime. Nature 464, 1324-1328 (2010).

31. Even, U. The Even-Lavie valve as a source for high intensity supersonic beam. EPJ Tech. Instrum. 2, 17 (2015).

32. Perreault, W. E., Mukherjee, N. \& Zare, R. N. Stark-induced adiabatic Raman passage examined through the preparation of D2 $(\mathrm{v}=2, \mathrm{j}=0)$ and D2 $(\mathrm{v}=2, \mathrm{j}=2, \mathrm{~m}=0) . J$. 
Chem. Phys. 150, 234201 (2019).

33. Mukherjee, N. \& Zare, R. N. Stark-induced adiabatic Raman passage for preparing polarized molecules. J. Chem. Phys. 135, 024201 (2011).

34. Mukherjee, N., Perreault, W. E. \& Zare, R. N. Stark-Induced Adiabatic Passage Processes to Selectively Prepare Vibrationally Excited Single and Superposition of Quantum States. in Frontiers and Advances in Molecular Spectroscopy 1-46 (Elsevier Inc., 2018).

35. Perreault, W. E., Zhou, H., Mukherjee, N. \& Zare, R. N. Harnessing the power of adiabatic curve crossing to populate the highly vibrationally excited $\mathrm{H}_{2}(v=7, j=0)$ level. Phys. Rev. Lett. 124, 163202 (2020).

36. Mukherjee, N., Dong, W. \& Zare, R. N. Coherent superposition of M-states in a single rovibrational level of $\mathrm{H}_{2}$ by Stark-induced adiabatic Raman passage. J. Chem. Phys. 140, 074201 (2014).

37. Diep, P. \& Johnson, J. K. An accurate $\mathrm{H}_{2}-\mathrm{H}_{2}$ interaction potential from first principles. $J$. Chem. Phys. 112, 4465-4473 (2000).

38. Buck, U. Rotationally Inelastic Scattering of Hydrogen Molecules and the Non-spherical Interaction. Faraday Discuss. Chem. Soc. 73, 187-203 (1982).

39. Buck, U., Huisken, F., Kohlhase, A., Otten, D. \& Schaefer, J. State resolved rotational excitation in $\mathrm{D}_{2}+\mathrm{H}_{2}$ collisions. J Chem Phys 78, 4439-4450 (1983).

40. Croft, J. F. E., Balakrishnan, N., Huang, M. \& Guo, H. Unraveling the Stereodynamics of Cold Controlled HD- H2 Collisions. Phys. Rev. Lett. 121, 113401 (2018).

41. Hu, M. G. et al. Direct observation of ultracold bimolecular reactions. Science 366, 11111115 (2019).

42. Perreault, W. E., Mukherjee, N. \& Zare, R. N. Angular and internal state distributions of $\mathrm{H}_{2}{ }^{+}$generated by $(2+1)$ resonance enhanced multiphoton ionization of $\mathrm{H} 2$ using time-offlight mass spectrometry. J. Chem. Phys. 144, 214201 (2016). 


\section{Figures}

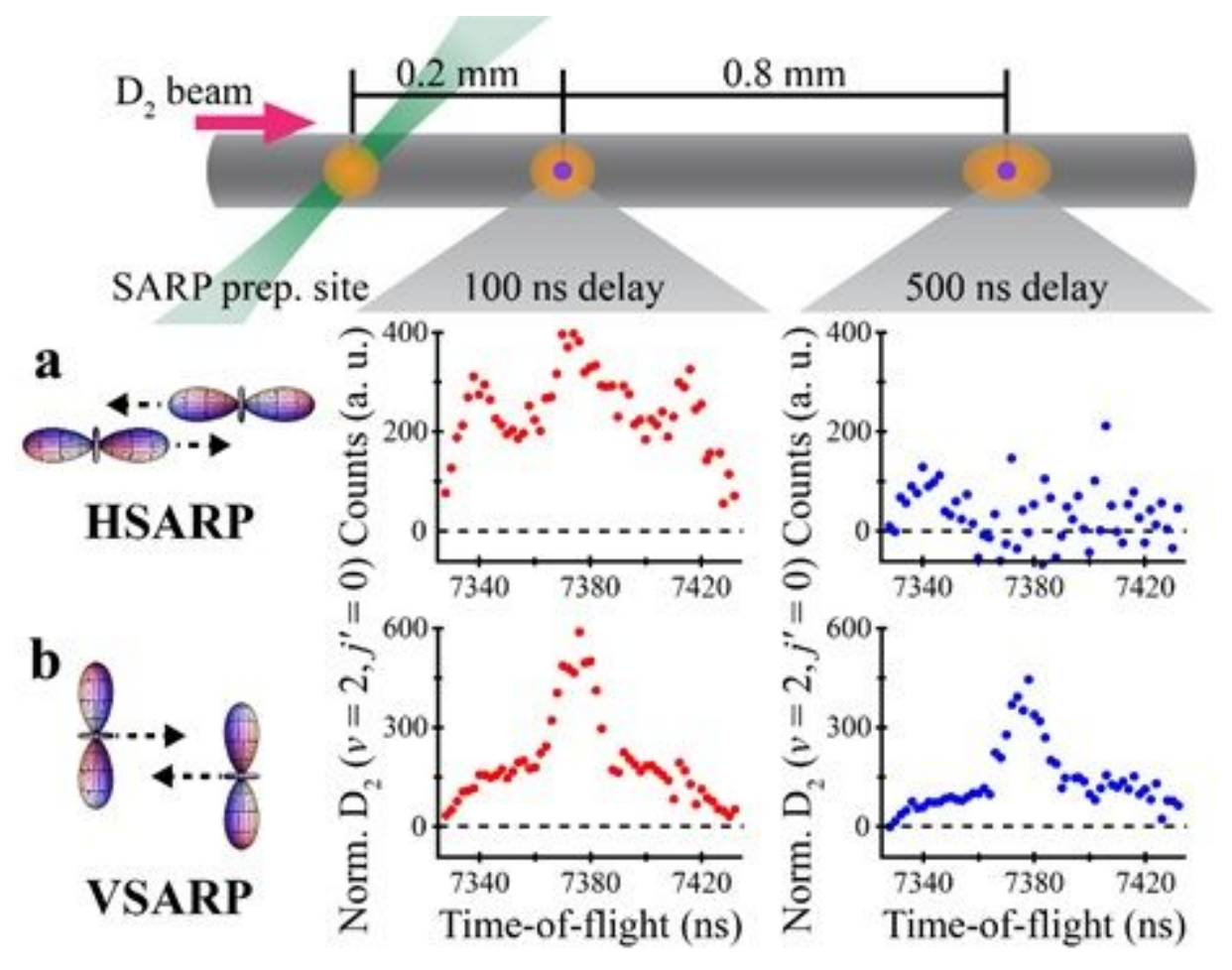

Figure 1

Measured time-of-flight distributions of scattered D2 $\left(v=2, j^{\prime}=0\right)$ for the HSARP (a) and VSARP (b) alignments at delays of $100 \mathrm{~ns}$ (red dots) and $500 \mathrm{~ns}$ (blue dots) after SARP preparation. In HSARP, the total number of counts drops by $\sim 90 \%$, while for VSARP it drops by only $\sim 40 \%$. (See Table I) The negative points for HSARP at $500 \mathrm{~ns}$ result from the signal level falling below the stochastic fluctuations of the background noise and should be taken only as a demonstration of the dramatic reduction in relaxation rate. Schematic at top shows the SARP preparation of a defined region of the molecular beam (orange spot). As the delay is increased, this spot propagates downstream and spreads out. As described in the text, only those scattering products within the small REMPI volume (purple spots) are detected 


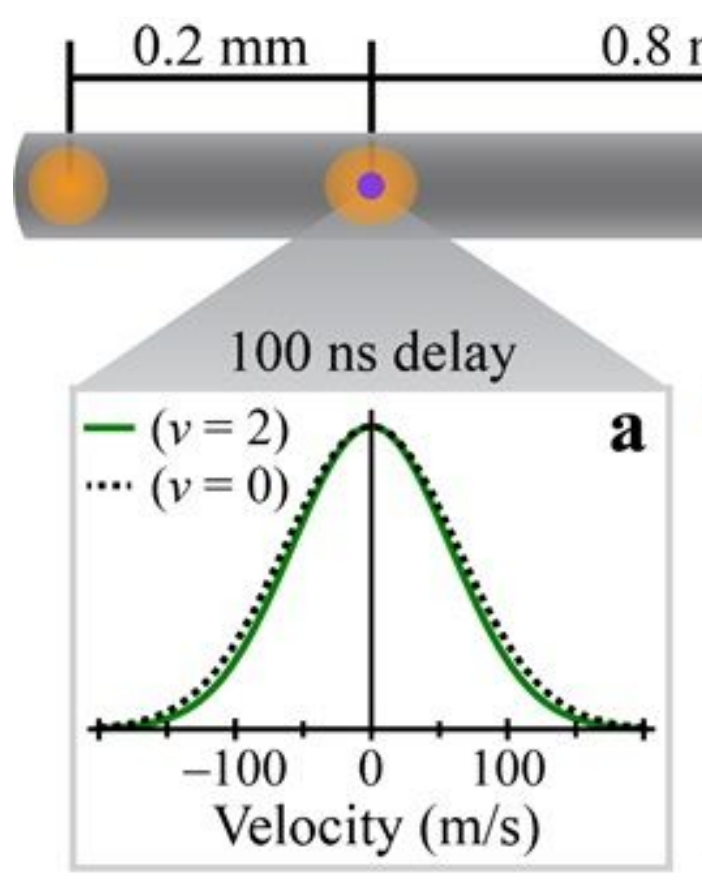

\section{0 ns delay}

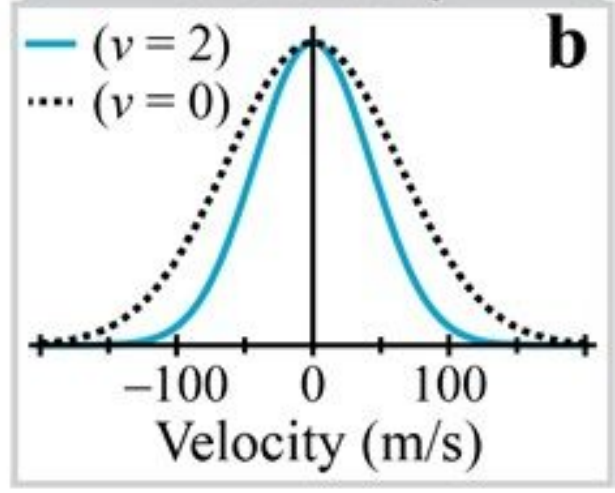

$$
\begin{aligned}
& -(v=2) /(v=2) 100 \mathrm{~ns} \\
& --(v=2) /(v=0) 100 \mathrm{~ns} \\
& -(v=2) /(v=2) 500 \mathrm{~ns} \\
& --(v=2) /(v=0) 500 \mathrm{~ns}
\end{aligned}
$$

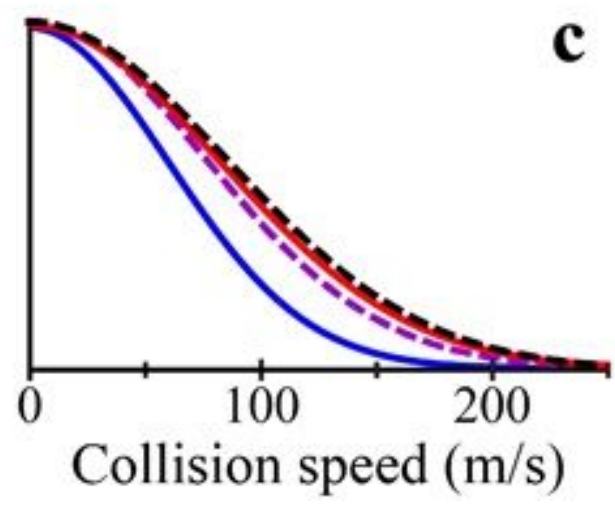

\section{Figure 2}

(a) The velocity distributions of the prepared D2 $(v=2, j=2)$ (green curve) and unprepared D2 $(v=0, j=1$, 2) (black dotted curve) at 100 ns delay. (b) The velocity distributions of the prepared $D 2(v=2, j=2)$ (cyan curve) and unprepared D2 $(v=0, j=1,2)$ (black dotted curve) at 500 ns delay. All velocities are determined by fitting the time-of-flight distribution taking into account the electron recoil, which gives a measurement error of less than $3 \mathrm{~m} / \mathrm{s}$. (c) Collision speeds for both the aligned-aligned (red and blue curves) and aligned-unaligned (black and magenta dashed curves) collision pairs at the two delays. As described in the Methods section, these curves are generated by convoluting the velocity distributions given in (a) and (b). The only substantial change is in the collision speed for the aligned-aligned collisions at $500 \mathrm{~ns}$ (solid blue curve). All three other curves are within our measurement error. 


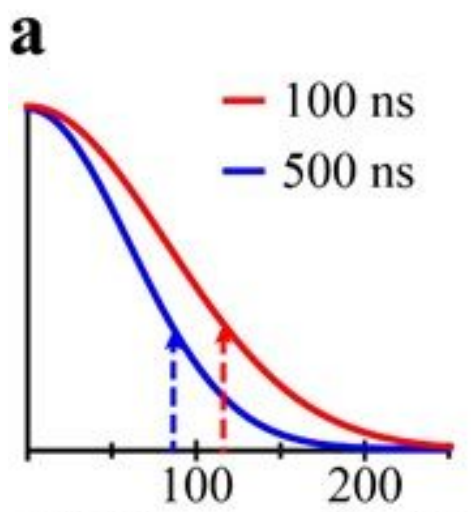

Collision speed $(\mathrm{m} / \mathrm{s})$

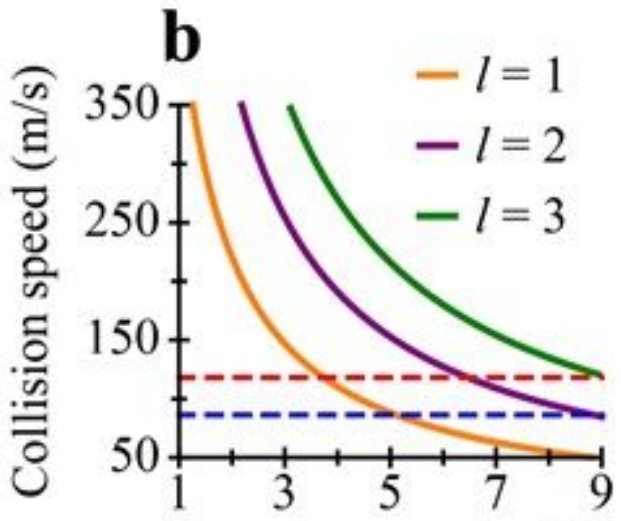

Impact parameter $(\AA)$

\section{Figure 3}

(a) The collision speed distribution for the D2 $(v=2) / D 2(v=2)$ collision pair at $100 \mathrm{~ns}$ (solid red) and 500 ns delay (solid blue). The red and blue dashed lines show the collision velocity range containing $85 \%$ of the D2 ( $(v)$ )/ D2 ( $(v=2)$ collisions at 100 and 500 ns delays, respectively. (b) Contour lines for the orbital angular momenta I = 1 (solid orange), I = 2 (solid purple), I = 3 (solid green) as a function of the collision speed and impact parameter. The red and blue dashed lines give the upper limit of the collision speed range for the delays of 100 ns and 500 ns corresponding to the dashed lines in (a).
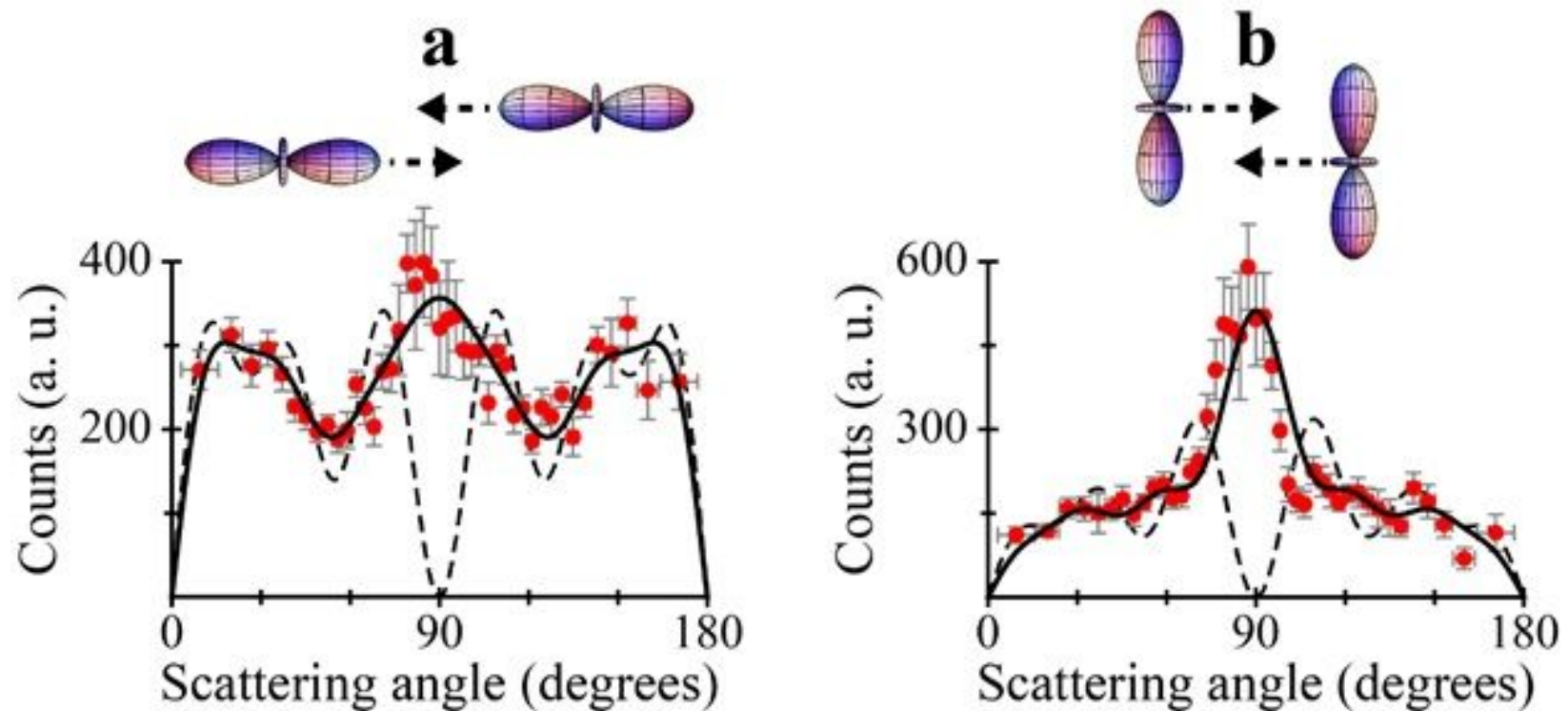

Figure 4

Experimentally measured scattering angular distributions and fits using partial wave analysis for HSARP (a) and VSARP (b) bond-axis alignments. The red dots represent experimental data taken at 100 ns delay. The solid and dashed black curves represent fits using partial wave analysis. As described in the main text, the solid black curve gives the fit produced using even outgoing orbitals $I^{\prime}=0,2,4,6$, whereas the dashed curve gives the fit produced using odd outgoing orbitals l' $=1,3,5$. The error bars represent the standard deviation in each time-of-flight bin. 